\title{
On the kinetics of suicide substrates
}

\author{
Meghan A. Burke ${ }^{a}$, P.K. Maini ${ }^{b}$ and J.D. Murray ${ }^{\text {a.c }}$ \\ 'Centre for Mathematical Biology, Mathematical Institute, 24-29 St. Giles', Oxford OXI 3LB, U.K., 'Department of Mathematics, \\ University of Utah, Salt Lake City, UT 84112 and 'Applied Mathematics Department, FS-20, University of Washington, Seattle, WA \\ 98195, U.S.A.
}

Received 10 January 1990

Accepted 5 February 1990

Suicide substrate; Enzyme kinetic model; Approximate solution technique; Enzyme targeting

We consider a realistic suicide substrate reaction which can be represented by four rate equations for the concentrations of the various molecules as functions of time. We present a general procedure to obtain accurate, approximate solutions analytically in terms of the rate equation parameters. This systematic technique provides more accurate approximations to the exact (numerical) solutions than other approximate methods which have been proposed based on a pseudo-steady state hypothesis.

\section{Introduction}

An enzyme system of considerable experimental interest [1-3] is the mechanism-based inhibitor, or 'suicide substrate' system, represented by [4]

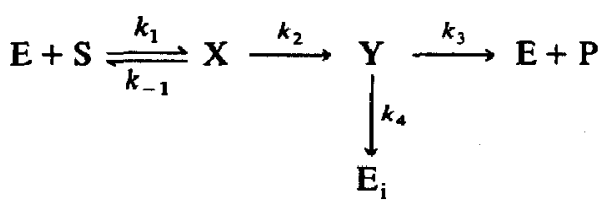

where $E, S$ and $P$ denote enzyme, substrate and product, respectively, $\mathrm{X}$ and $\mathrm{Y}$ enyme-substrate intermediates, $\mathrm{E}_{\mathrm{i}}$ inactivated enzyme, and the $k \mathrm{~s}$ are positive rate constants. In this system, $Y$ can follow one of two pathways, namely, to $E+P$ with rate $k_{3}$ or to $\mathrm{E}_{\mathrm{i}}$ with rate $k_{4}$. The ratio of these rates, $k_{3} / k_{4}$, is called the partition ratio and is denoted by $r$. Both of these pathways are considered to be irreversible over the time scale of the reaction [5]. $S$ is known as a suicide substrate because it binds to the active site of an enzyme -

Correspondence address: M.A. Burke, Centre for Mathematical Biology, Mathematical Institute, 24-29 St. Giles', Oxford OX1 3LB, U.K. like a substrate - but the enzyme converts it into an inhibitor which irreversibly inactivates the enzyme. Thus, the enzyme 'commits suicide.'

Suicide substrates are important because they provide a way to target a specific enzyme for inactivation. They are especially useful in drug administration, since they are not harmful in their common form and only the particular targeted enzyme can convert them to their inhibitor form. For example, suicide substrates have been investigated for use in the treatment of depression (monoamine oxidase inhibitors [2]), epilepsy (brain GABA transaminase inhibitors (31), and some tumors (ornithine decarboxylase inhibitors [2]).

Suicide substrate kinetics have been considered by Waley [5] and by Tatsunami et al. [6]. Waley proposed that the factor which determined whether the substrate is exhausted before all the enzyme is inactivated is $r \mu$, where $\mu$ is the ratio of the initial concentration of enzyme to that of substrate, namely, $E_{0} / S_{0}$. Tatsunami et al, on the other hand, found the determining factor to be $(1+r) \mu$. When $(1+r) \mu>1$ the substrate is exhausted, while for $(1+r) \mu<1$, all the enzyme is inactivated. (When $(1+r) \mu=1$, both occur.) While it is true that the results obtained by Tatsunami et 
al. were more consistent with numerical solutions of the full rate equations obtained from the system (eq. 1) than were Waley's, both results deviate from the solution when $\mu$ approaches 1 . This highlights a shortcoming that occurs in most pseudo-steady-state approximations for enzyme kinetics: their validity decreases for increasing values of $E_{0} / S_{0}$. Thus, a new analysis is needed which is valid when the concentrations $E_{0}$ and $S_{0}$ are of the same order of magnitude or $E_{0}$ is very. much larger than $S_{0}$, i.e., $E_{0} / S_{0}$ is not small [7]. Ideally, we would like an analytical, uniformly valid solution for all time. Such a solution clearly shows the transition from the fast-transient phase to the pseudo-steady-state phase. It also provides quantitative expressions for the time evolution of the reaction in terms of the parameters (which are related to the rate constants). This paper shows how to derive such solutions.

First, we set up the rate equations using the Law of Mass Action. We examine the equations in the initial stages of the reaction and also when the system is effectively at a pseudo-steady state. We are required to choose a time scale - the 'inner' time scale - that facilitates the analysis of the initial, fast transient. As the system approaches pseudo-steady state, we must choose a new time scale - the 'outer' time scale - to facilitate the study of the long-time evolution of the reactants. We then solve these two sets of equations for different sets of parameter values, and match the inner and outer solutions to obtain a uniformly valid approximation to the solution of the full system. Finally, we compare this approximation to previous ones, and to numerical solutions of the original rate equations.

\section{Rate equations}

The rate equations obtained from eq. 1 using the Law of Mass Action are given by:

$$
\begin{aligned}
& \frac{\mathrm{d}[\mathrm{S}]}{\mathrm{d} t}=-k_{1}[\mathrm{E}][\mathrm{S}]+k_{-1}[\mathrm{X}] \\
& \frac{\mathrm{d}[\mathrm{E}]}{\mathrm{d} t}=-k_{1}[\mathrm{E}][\mathrm{S}]+k_{-1}[\mathrm{X}]+k_{3}[\mathrm{Y}]
\end{aligned}
$$

$$
\begin{aligned}
& \frac{\mathrm{d}[\mathrm{X}]}{\mathrm{d} t}=k_{1}[\mathrm{E}][\mathrm{S}]-k_{-1}[\mathrm{X}]-k_{2}[\mathrm{X}] \\
& \frac{\mathrm{d}[\mathrm{Y}]}{\mathrm{d} t}=k_{2}[\mathrm{X}]-k_{3}[\mathrm{Y}]-k_{4}[\mathrm{Y}] \\
& \frac{\mathrm{d}\left[\mathrm{E}_{\mathrm{i}}\right]}{\mathrm{d} t}=k_{4}[\mathrm{Y}] \\
& \frac{\mathrm{d}[\mathrm{P}]}{\mathrm{d} t}=k_{3}[\mathrm{Y}] .
\end{aligned}
$$

where [ ] denotes concentration, and $t$ time. Typical experimental initial conditions which complete the mathematical formulation are

$$
\begin{aligned}
& E(0)=E_{0}, S(0)=S_{0}, \\
& X(0)=Y(0)=E_{\mathrm{i}}(0)=P(0)=0 .
\end{aligned}
$$

Eq. 7 can be uncoupled from the rest, because [P] does not appear in any of the other equations; [P] can be evaluated by integration after $[Y]$ has been found.

The order of the system can be further reduced by noting that (adding eqs 3-6)

$$
\begin{aligned}
& \frac{\mathrm{d}}{\mathrm{d} t}\left([\mathrm{E}]+[\mathrm{X}]+[\mathrm{Y}]+\left[\mathrm{E}_{\mathrm{i}}\right]\right\}=0 \\
& \Rightarrow[\mathrm{E}]+[\mathrm{X}]+[\mathrm{Y}]+\left[\mathrm{E}_{\mathrm{i}}\right]=E_{0} .
\end{aligned}
$$

That is, the total amount of enzyme, whether free, inactive or in a complex, remains constant. Using eq. 10 to eliminate $[E]$, we obtain the reduced system

$$
\begin{aligned}
\frac{\mathrm{d}[\mathrm{S}]}{\mathrm{d} t}= & -k_{1}\left(\mathrm{E}_{0}-[\mathrm{X}]-[\mathrm{Y}]-\left[\mathrm{E}_{\mathrm{i}}\right]\right)[\mathrm{S}] \\
& +k_{-1}[\mathrm{X}] \\
\frac{\mathrm{d}[\mathrm{X}]}{\mathrm{d} t}= & k_{1}\left(\mathrm{E}_{0}-[\mathrm{X}]-[\mathrm{Y}]-\left[\mathrm{E}_{\mathrm{i}}\right]\right)[\mathrm{S}] \\
& -\left(k_{-1}+k_{2}\right)[\mathrm{X}] \\
\frac{\mathrm{d}[\mathrm{Y}]}{\mathrm{d} t}= & k_{2}[\mathrm{X}]-\left(k_{3}+k_{4}\right)[\mathrm{Y}] \\
\frac{\mathrm{d}\left[\mathrm{E}_{\mathrm{i}}\right]}{\mathrm{d} t}= & k_{4}[\mathrm{Y}]
\end{aligned}
$$

This is the system specifically considered by Tatsunami et al. [6]. 


\section{Model system in nondimensional form}

It is always advantageous to express the model system in nondimensional terms. By converting to nondimensional variables and parameters, we can then consider quantities on an absolute scale, independent of specific units. Such a procedure also immediately gives a measure of the relative magnitudes of the various contributions to the rate equations.

There are several ways to nondimensionalise the system. Here we follow the procedure of Segel and Slemrod [8] (see also refs. 9 and 10) and nondimensionalise the rate equations (eqs 11-14) using two different time scales related to the fasttransient phase (inner time scale) and the pseudosteady-state phase (outer time scale). The inner equations describe the system during the early stage of the reaction, when [S] has not decreased significantly, and $[X]$ and $[Y]$ are small, but growing.

We nondimensionalise our variables by setting

$[S]=S_{0} s, \quad[X]=\frac{E_{0} S_{0}}{S_{0}+K} x$,

$[\mathrm{Y}]=E_{\mathrm{o}} y, \quad\left[\mathrm{E}_{\mathrm{i}}\right]=E_{\mathrm{u}} e_{\mathrm{i}}$,

where

$K=\frac{k_{-1}+k_{2}}{k_{1}}$

and $s, x, y$ and $e_{\mathrm{i}}$ are now dimensionless variables. The fast-transient time scale is taken as

$\tau=t / t_{\mathrm{p}}=t k_{1}\left(S_{0}+K\right)$

and the pseudo-steady state time scale as

$T=t / t_{s}=t \epsilon\left(k_{-1}+k_{2}\right)$

with

$\epsilon=\frac{E_{0}}{S_{0}+K}$.

Using the scalings in eq. 15 with $\tau$ as the time scale, we obtain the nondimensionalised versions of eqs 11-14 for the fast-transient phase, namely

$$
\begin{aligned}
\frac{\mathrm{d} s}{\mathrm{~d} \tau}= & \epsilon\left[-s+\frac{\sigma}{1+\sigma} s x+s y+s e_{\mathrm{i}}\right. \\
& \left.+\frac{\rho}{(1+\rho)(1+\sigma)} x\right]
\end{aligned}
$$

$$
\begin{aligned}
& \frac{\mathrm{d} x}{\mathrm{~d} \tau}=s-\left(\frac{\sigma}{1+\sigma}\right) s x-s y-s e_{\mathrm{i}}-\frac{x}{1+\sigma} \\
& \frac{\mathrm{d} y}{\mathrm{~d} \tau}=\left(\frac{\sigma}{(1+\sigma)^{2}(1+\rho)}\right) x-\left(\frac{\psi}{(1+\sigma)}\right) y \\
& \frac{\mathrm{d} e_{\mathrm{i}}}{\mathrm{d} \tau}=\left(\frac{\phi}{1+\sigma}\right) y
\end{aligned}
$$

where

$$
\begin{aligned}
& \sigma=\frac{S_{0}}{K}, \quad \rho=\frac{k_{-1}}{k_{2}}, \quad \psi=\frac{k_{3}+k_{4}}{k_{-1}+k_{2}}, \\
& \phi=\frac{k_{4}}{k_{-1}+k_{2}} .
\end{aligned}
$$

The initial conditions (eq. 8) now become, on using eq. 15 ,

$s(0)=1, \quad x(0)=0, \quad y(0)=0, \quad e_{\mathrm{i}}(0)=0 .(25)$

Note that $\epsilon$ small in eq. 20 implies that $s$ changes little, while $x$ and $y$ change significantly during the fast-transient phase. An important part of the analysis is quantifying these statements. By saying ' $s$ has not changed significantly,' we mean that the nondimensional time derivative of $s$ in our equations is not zero, but is equal to an expression which is multiplied by a small parameter, $\epsilon$. We refer to the right-hand side of eq. 20 as being 'of order $\epsilon$; or $O(\epsilon)$. Conversely, since $x$ and $y$ are changing significantly, we say their time derivatives are 'of order 1,' or $O(1)$ - they are not multiplied by $c$. We use this approach when solving the equations by equating coefficients of powers of $\epsilon$. Since $E_{0} /\left(S_{0}+K\right)$ is our small parameter, we can extend the validity of the traditional quasi-steady-state assumption (QSSA) to cases where $E_{0} / S_{0}$ is not small.

The same constants and scalings are used for the dependent variables but with $T$ as the time scale to nondimensionalise the rate equations for the pseudo-steady-state phase:

$$
\begin{aligned}
\frac{\mathrm{d} s}{\mathrm{~d} T}= & -s\left[(\sigma+1)-\sigma x-(\sigma+1) y-(\sigma+1) e_{\mathrm{i}}\right] \\
& +\frac{\rho}{1+\rho} x \\
\epsilon \frac{\mathrm{d} x}{\mathrm{~d} T}= & s\left[(\sigma+1)-\sigma x-(\sigma+1) y-(\sigma+1) e_{\mathrm{i}}\right] \\
& -x
\end{aligned}
$$


$c \frac{\mathrm{d} y}{\mathrm{~d} T}=\left(\frac{\sigma}{(1+\sigma)(1+\rho)}\right) x-\psi y$

$\epsilon \frac{\mathrm{d} e_{\mathrm{i}}}{\mathrm{d} T}=\phi y$

where $\epsilon, \sigma, \rho, \psi$ and $\phi$ are given by eq. 24 .

Now that we have the two sets of dimensionless rate equations for the two time periods, we find uniformly valid approximate solutions by asymptotic methods. In the following section, we simply describe the procedure heuristically and present the solutions.

\section{Solution method and solutions}

The fact that a small parameter $0<\epsilon \ll 1 \mathrm{mul}$ tiplies the derivatives in eqs $27-29$ indicates that this is a singular perturbation problem. One class of such problems is immediately recognised if, on setting $\epsilon=0$, the order of the system of differential equations is reduced: such a reduced system cannot in general satisfy all the initial conditions. Singular perturbation techniques are very important and are powerful for determining asymptotic solutions of such systems of equations for small e. Asymptotic solutions are usually remarkably accurate approximations to the exact solutions. A practical and elementary discussion of some of the key techniques is given in Murray's book on asymptotic analysis [11]. In this section, the philosophy and actual singular perturbation technique will be described in detail and the asymptotic solutions to eqs $20-23$ and 26-29 for $0<\epsilon \ll 1$ derived. The main reason for doing this is to indicate when we can neglect the $\epsilon$ terms in practical situations [12].

If we set $\epsilon=0$, the right-hand sides of eqs 27-29 give $x, y$ and $e_{\mathrm{i}}$ in terms of $s$. This is what we mean when we say that the system is in pseudo-steady state. In the fast-transient phase, $s$ is approximately constant and $x, y$ and $e_{\mathrm{i}}$ change. Therefore, we solve the inner equations, eqs 20-23 (which are valid for small $t$ ), with the initial conditions, eq. 25 . For $t$ large, we solve the system, eqs 26-29 (where $T$ is $O(1)$ ), i.e., the pseudo-steady-state system, and match it to the solution for $t$ of $O(1)$. So, an appropriate time scale for $t$ large is proportional to $\epsilon t$ rather than $t$. We analyse eqs $20-23$ near $t=0$, i.e., $\tau=0$, after which we shall obtain the solutions to eqs 26-29 for large $t$ and finally show how to determine a uniformly valid solution for all $t \geq 0$ [12].

\subsection{Inner or singular solutions}

We begin with the fast-transient phase equations, eqs 20-23, with initial conditions, eq. 25 , and because of the presence of the small $\epsilon$ we look for a Taylor series solution in the form

$s(\tau)=s^{(0)}(\tau)+\epsilon s^{(1)}(\tau)+\epsilon^{2} s^{(2)}(\tau)+\ldots$

for each of the variables $s, x, y$ and $e_{\mathrm{i}}$. Substituting these into eqs 20-23 and equating like powers of $\epsilon$, we can find the first terms in the series. For example, substituting the series solutions like eq. 30 into eq. 20 and equating terms of $O(1)$ gives $\mathrm{d} s^{(0)} / \mathrm{d} \tau=0$. Since $s(0)=1$, this implies that $s^{(0)}(\tau) \equiv 1$. That is, $s$ is approximately constant in the fast-transient phase. Similarly, eq. 22 gives

$\frac{\mathrm{d} y^{(0)}}{\mathrm{d} \tau}=-\frac{\psi}{1+\sigma} y^{(0)}$.

The only solution to this equation which fits the initial condition $y(0)=0$ is $y^{(0)}(\tau) \equiv 0$. In the same way, eq. 23 yields

$\frac{\mathrm{d} e_{\mathrm{i}}^{(0)}}{\mathrm{d} \tau}=\frac{\phi}{1+\sigma} y^{(0)}=0$

which implies that $e_{\mathrm{i}}^{(0)}(\tau) \equiv 0$ since $e_{\mathrm{i}}(0)=0$. Finally, substituting the series solutions into eq. 23 , we obtain

$$
\begin{aligned}
\frac{\mathrm{d} x^{(0)}}{\mathrm{d} \tau}= & s^{(0)}-s^{(0)} y^{(0)}-s^{(0)} e_{\mathrm{i}}^{(0)} \\
& -\frac{x^{(0)}}{1+\sigma}-\frac{\sigma s^{(0)} x^{(0)}}{1+\sigma} .
\end{aligned}
$$

With the above solutions for $s^{(0)}, y^{(0)}$ and $e_{i}^{(0)}$, this becomes

$\frac{\mathrm{d} x^{(0)}}{\mathrm{d} \tau}=1-x^{(0)}$

which is easily solved with $x(0)=0$ to give $x^{(0)}(\tau)$ $=1-e^{-\tau}$. 
To obtain nonzero solutions for $y$ and $e_{\mathrm{i}}$, we need to determine $y^{(1)}(\tau)$ and $e_{i}^{(1)}(\tau)$. This involves matching the coefficients of $O(\epsilon)$ terms.

We note that

$\epsilon=\frac{E_{0}}{S_{0}\left(1+K / S_{0}\right)}=\frac{E_{0}}{S_{0}} \frac{\sigma}{1+\sigma}$

which implies that

$\sigma=\left(\frac{S_{0}}{E_{0}}\right) \epsilon+O\left(\epsilon^{2}\right)$.

Since we are assuming $S_{0} / E_{0}=O(1)$, this implies that $\sigma=O(\epsilon)$ [9]. Here we introduce a similarity variable for $\sigma$,

$\boldsymbol{\sigma}=\boldsymbol{\epsilon} \boldsymbol{p}$

where $p$ is a constant of $O(1)$. We show the $\varepsilon$ factor explicitly so that we can match it with the $O(\epsilon)$ terms. Substituting eq. 37 for $\sigma$ in eq. 22, we equate terms of $O(\epsilon)$ :

$\frac{\mathrm{d} y^{(1)}}{\mathrm{d} \tau}=\frac{p}{(1+\rho)} x^{(0)}-\psi y^{(1)}$.

Since we already have an expression for $x^{(0)}$, we can solve the above linear equation for $y^{(1)}(\tau)$ :

$y^{(1)}(\tau)=\frac{p}{\psi(1+\rho)}\left(\frac{1-e^{-\psi \tau}}{\psi}+\frac{e^{-\psi \tau}-e^{-\tau}}{\psi-1}\right)$.

Now, matching coefficients in eq. 23 yields an equation for $\mathrm{d} e_{\mathrm{i}} / \mathrm{d} \tau$ in terms of $y^{(1)}$ which can be solved to give

$e_{i}^{(1)}(\tau)=\frac{\phi p}{(1+\rho)}\left(\frac{\tau}{\psi}+\frac{e^{-\tau}-1}{\psi-1}+\frac{1-e^{-\psi \tau}}{\psi^{2}(\psi-1)}\right)$.

Note that in obtaining $e_{i}^{(1)}(\tau)$, we assumed $\phi=O(1)$. If it were the case that $\phi=O(\epsilon)$, we would have used another similarity variable, $q=$ $\epsilon \phi$, and found that $e_{i}^{(1)}(\tau)=0$, but that $e_{i}^{(2)}(\tau)$ gives the same result as $e_{i}^{(1)}(\tau)$ above.

In a similar manner, we can find the coefficients of higher order terms in the series. For example, the $O(\epsilon)$ terms of eq. 20 give

$s^{(1)}(\tau)=-\frac{\tau}{1+\rho}+\frac{\rho}{1+\rho}\left(e^{-\tau}-1\right)$.

Note that all of these solutions satisfy the initial conditions, eq. 25 .

\subsection{Outer or pseudo-steady-state solutions}

We now proceed to look for solutions to the pseudo-steady-state problem in the same way with a view to matching the two time period solutions. Recall that these solutions will not in general satisfy the initial conditions. Undetermined constants of integration are evaluated by matching the solution domains together.

As before, we look for simple solutions to eqs $26-29$ in the form

$s(T)=s_{(0)}(T)=\epsilon s_{(1)}(T)+\epsilon^{2} s_{(2)}(T)+\ldots$

for each of the substance variables $s, x, y$ and $e_{\mathrm{i}}$. After we have substituted these solutions into eqs 26-29, we once again equate coefficients of powers of $\epsilon$. Here, however, we must solve for the undetermined constants of integration, which we do by the method of matched asymptotic expansions; i.e., the inner solution as $\tau \rightarrow \infty$ must match the outer solution as $T \rightarrow 0$ (see ref. 11).

Taking the $O(1)$ terms, we find

$0=s_{(0)}-s_{(0)} y_{(0)}-x_{(0)}-s_{(0)} e_{\mathrm{i}(0)}$

from eq. 27, and, assuming $\psi=O(1), y_{(0)}=0$ from eq. 28. Together, these give

$x_{(0)}=s_{(0)}\left(1-e_{\mathrm{i}(0)}\right)$.

Similarly, we obtain

$y_{(1)}=\frac{p}{\psi(1+\rho)} x_{(0)}$.

In order to equate coefficients further, we need to determine the order of magnitude of each of the terms. Experimentally, we know that there are two fundamentally different outcomes: either all of the substrate is exhausted, or all of the enzyme is inactivated. These correspond to $\phi=O(1)$ with $\psi=O(1)$, and $\psi=O(1)$ with $\phi=O(\epsilon)$ (refer to eq. 
24 for the parameter relations). We solve the equations for each of these sets of constraints.

Case 1: $\rho=O(1), \psi=O(1), \phi=O(1)$

This is the case when all of the rate constants are of the same order of magnitude. By assuming $\phi=O(1)$, we solve to obtain

$s_{(0)}(T)=\frac{1-\beta}{1-C e^{T[1-(1 / \beta) /(1+\rho)}}$

$c_{\mathrm{i}(0)}(T)=\frac{1-s_{(0)}(T)}{\beta}$

where

$\beta=\frac{\psi}{\phi p}$

and $C$ is a constant of integration. We find a value for $C$ by matching the values of $s_{(0)}(T)$ as $T \rightarrow 0$ with the value of $s^{(0)}(\tau)$ as $\tau \rightarrow \infty$. We know $s^{(0)}(\tau) \equiv 1$, so $C=\beta$. (See ref. 11 for details of matching.)

Case 2: $\rho=O(1), \psi=O(1), \phi=O(\epsilon)$

Assuming $\phi=O(\epsilon)$ gives

$s_{(0)}(T)=e^{-T /(1+\rho)}$

$e_{\mathrm{i}(0)}=0$

$\epsilon \boldsymbol{E}_{\mathrm{i}(1)}(T)=\frac{1-e^{-T /(1+\rho)}}{\beta}$

where again we have matched with the inner solutions.

In both the inner and outer solutions, we could continue to solve for terms of higher order of $\epsilon$ in the series, eqs 30 and 42 . The solutions would become progressively more complicated, but in each case the equations are linear. The higher order terms also become progressively smaller; for most practical purposes, therefore, the first nonzero term is sufficiently accurate.

Now that we have solutions for the fast-transient and the pseudo-steady-state time periods, we can obtain composite solutions that are valid for all time $t \geq 0$ by a simple method detailed in ref. 13. We add the first term of the inner solutions to the corresponding term of the outer solutions and subtract their common part - the limit of the inner solution as time $(\tau)$ goes to infinity, which is the same as the limit of the outer solutions as time $(T)$ tends toward zero. For example, the inner solution for $s$ is $s^{(0)}(\tau)=1$. The outer solution for case 2 is $s_{(0)}=\exp (-T /(1+\rho))$. The limits described above are both 1 , so the composite solution is:

$$
\begin{aligned}
s_{\text {comp }}^{0} & =1+\exp \left(-\frac{T}{1+\rho}\right)-1 \\
& =\exp \left(-\frac{t}{t_{s}(1+p)}\right) .
\end{aligned}
$$

Doing the same for the other solutions, we obtain two sets of composite solutions, one for case 1 and one for case 2 , which are valid for all time.

Case 1:

$s_{\mathrm{comp}}^{0}(t)=\frac{1-\beta}{1-\beta e^{f(1-1 / \beta) / t_{\mathrm{s}}(1+\rho)}}$

$e_{\text {icomp }}^{0}(t)=\frac{1-s_{\text {comp }}^{0}}{\beta}$

$x_{\text {comp }}^{0}(t)=s_{\text {comp }}^{0}\left(1-e_{\text {icomp }}^{0}\right)-e^{-t / t_{p}}$

$y_{\text {comp }}^{0}(t)=0$

$$
\begin{aligned}
\epsilon y_{\text {comp }}^{1}(t)= & \frac{\sigma}{\psi(1+\rho)}\left(\frac{e^{-\psi t / t_{p}}-\psi e^{-t / t_{p}}}{\psi-1}\right. \\
& \left.+s_{\text {comp }}^{0}\left(1-e_{\text {comp }}^{0}\right)\right)
\end{aligned}
$$

Case 2:

$$
\begin{aligned}
& s_{\text {comp }}^{0}(t)=e^{-t / t_{\mathrm{s}}(1+\rho)} \\
& e_{\mathrm{icomp}}^{0}(t)=0 \\
& \epsilon e_{\mathrm{icomp}}^{1}(t)=\frac{1-s_{\mathrm{comp}}^{0}}{\beta} \\
& x_{\mathrm{comp}}^{0}(t)=s_{\mathrm{comp}}^{0}-e^{-t / t_{\mathrm{p}}} \\
& y_{\mathrm{comp}}^{0}(t)=0 \\
& \epsilon y_{\text {comp }}^{1}(t)=\frac{\sigma}{\psi(1+\rho)}\left(\frac{e^{-\psi t / t_{\mathrm{p}}}-\psi e^{-t / t_{\mathrm{p}}}}{\psi-1}+s_{\mathrm{comp}}^{0}\right)
\end{aligned}
$$


where $\beta=\psi / \phi p$.

Note that the important parameter distinguishing cases 1 and 2 is $\beta$. When $\beta<1$, case 1 holds, and when $\beta>1$, case 2 holds. This $\beta$ is, in fact, the same parameter that Tatsunami et al. [6] called $(1+r) \mu$. The above expressions show that for $\beta<1, e_{\mathrm{i}} \rightarrow 1$ as $T \rightarrow \infty$ (to first order in $\epsilon$ ), while for $\beta>1, s \rightarrow 0$ as $T \rightarrow \infty$ (to first order in $\epsilon$ ).
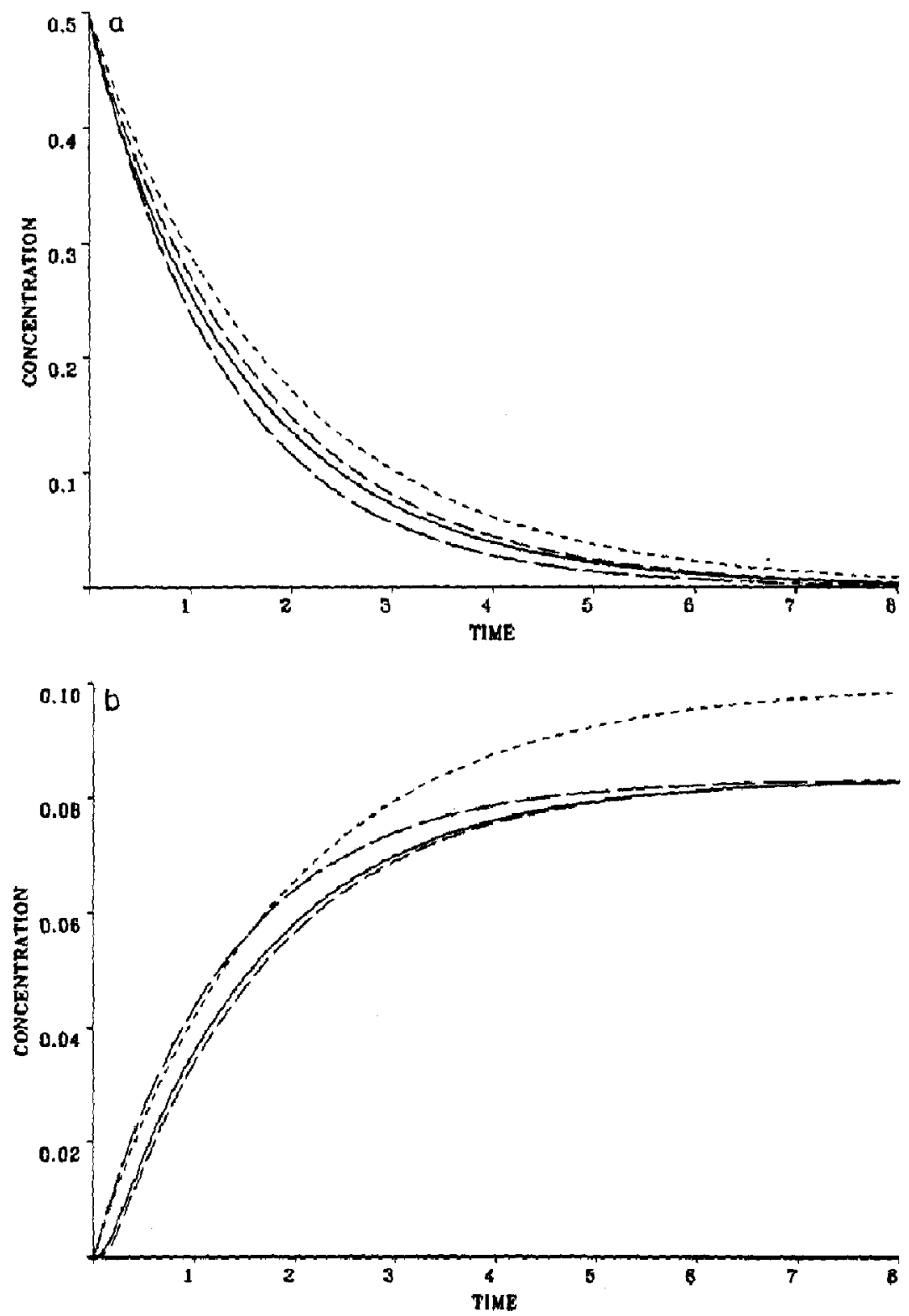

Fig. 1. Fitst two terms of the case 2 composite series solutions (-- ) compared to numerical solutions ( approximations: Walcy (-....) and Tatsumami et al. $(-\ldots \ldots)$ ( (a) Substrate cobcentration; (b) inactive enzyme concentration, Parameters: $k_{1}=2, k_{-1}=4, k_{2}=12, k_{3}=10, k_{4}=2, E_{0}=0.5, S_{0}=0.5$. 


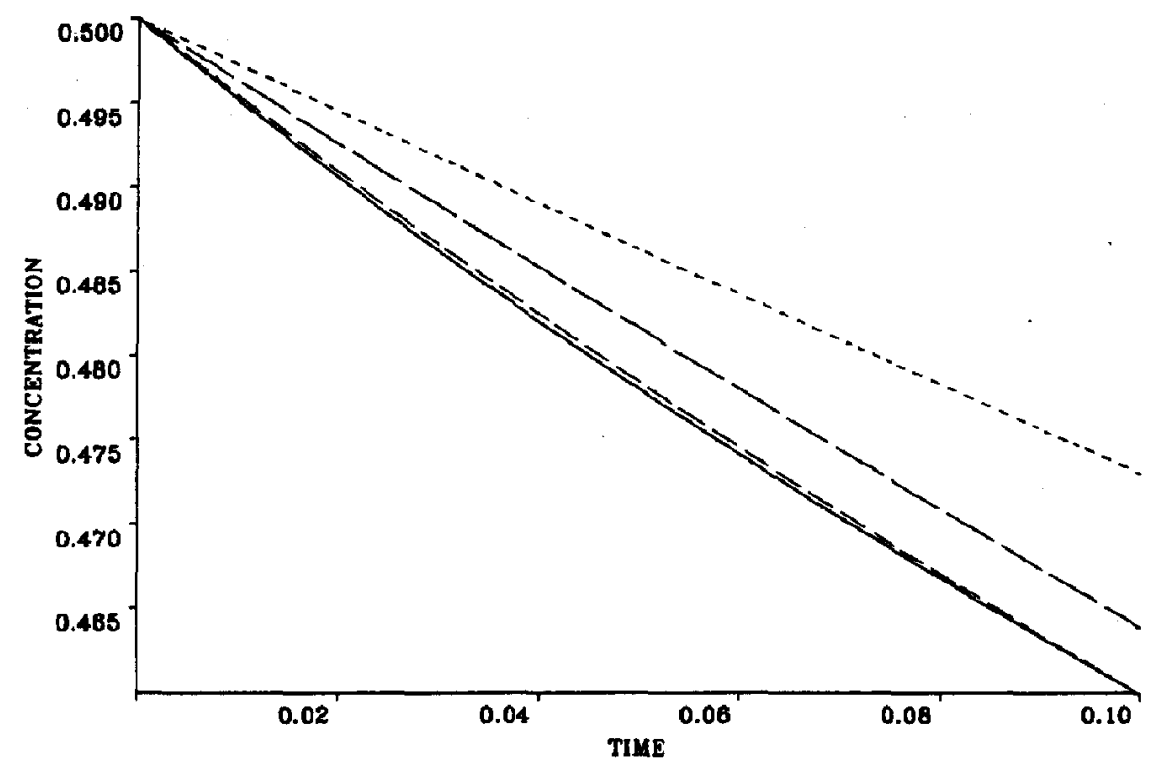

Fig. 2. Case 2 composite solutions $(---)$ compared to numerical solutions $(-\longrightarrow)$, Waley (-..---) and Tatsunami et al. $(---)$ approximations at small time for $S$. Parameters are the same as in fig. 1.

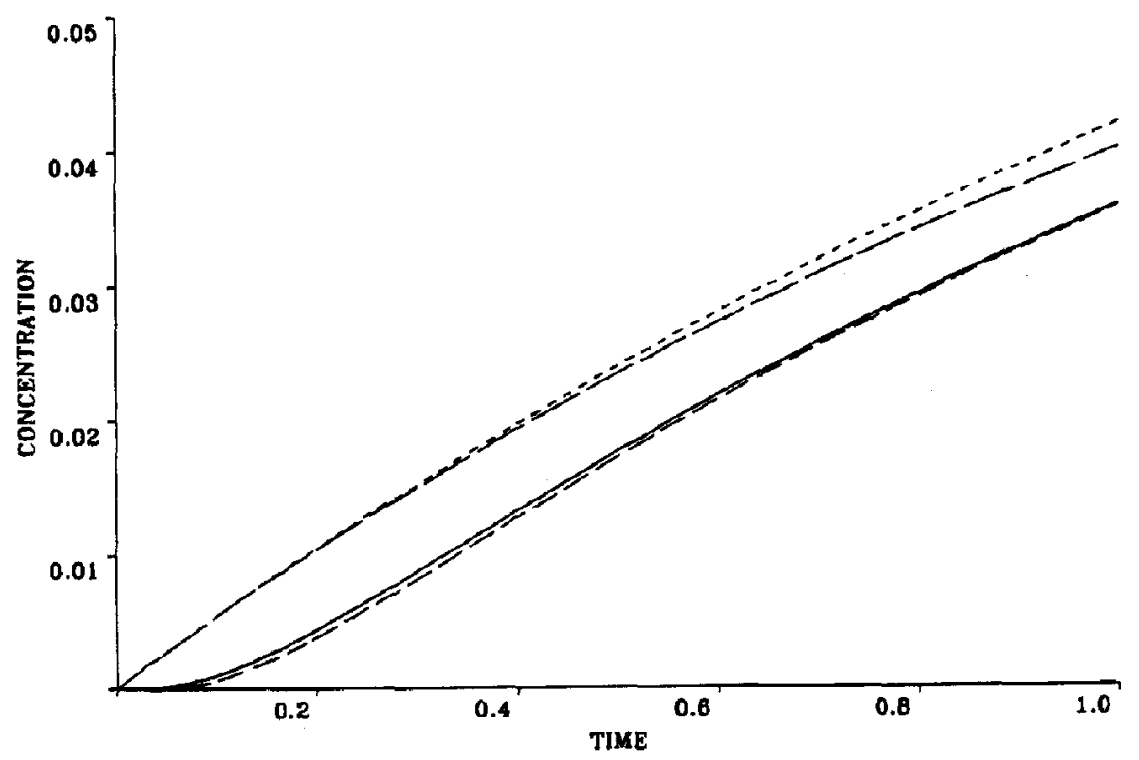

Fig. 3. Case 2 composite solutions (-- ) compared to numerical solutions ( $-\longrightarrow$ ), Waley (--.--) and Tatsunami et al. (- - -) approximations at small time for $E_{i}$. Parameters are the same as in fig. 1. 
These results agree with those of Tatsunami et al. [6].

Now that we have approximate asymptotic solutions to our nondimensionalised systems, we shall compare them to previous solutions and to numerical solutions to evaluate their accuracy.

\section{Numerical solutions and comparison with ana-} lytic solutions

To assess the accuracy of our analytic solutions, we compared results from our analysis with those obtained by solving the dimensional system, eqs 11-14, numerically. Since the numerical analysis was carried out on the dimensional system, the nondimensional concentrations were multiplied by their scale factors before plotting for ease of comparison. Previous authors [5,6] have been able to match only $S$ and $E_{\mathrm{i}}$ values with their models. With our procedure we can evaluate the intermediate concentrations as well.

The first two terms of the composite solutions are compared to previous and numerical solutions in fig. 1. We see that the present solutions are at least as accurate as previous approximations, and more accurate for most of the reaction.

Fig. 2 shows the approximations of Waley [5] and Tatsunami et al. [6] and numerical solutions compared to the composite solution for $S$ at small time (the inner domain). The case 2 parameters and solution set were used; the $O(\epsilon)$ terms were kept to give

$$
\begin{aligned}
s_{\mathrm{comp}} & =e^{-T /(1+\rho)}+\epsilon\left[\frac{\rho}{1+\rho} e^{t / t_{\mathrm{p}}}\right. \\
& +\frac{t}{t_{\mathrm{s}}} e^{t /\left(t_{\mathrm{s}}(1+\rho)\right)}\left(\frac{\rho}{(1+\rho)^{2}}+\frac{q p}{\psi(1+\rho)}\right) \\
& +e^{-2 t /\left(t_{\mathrm{s}}(1+\rho)\right)}\left(-p-\frac{p}{\psi(1+\rho)}+\frac{p q}{\psi}\right) \\
& \left.+e^{-t /\left(t_{\mathrm{s}}(1+\rho)\right)}\left(p+\frac{p}{\psi(1+\rho)}-\frac{p q}{\psi}-\frac{\rho}{1+\rho}\right)\right]
\end{aligned}
$$

Fig. 3 shows the previous and numerical solutions compared to the composite solution for $E_{\mathrm{i}}$ also at small time. Again, case 2 parameters and

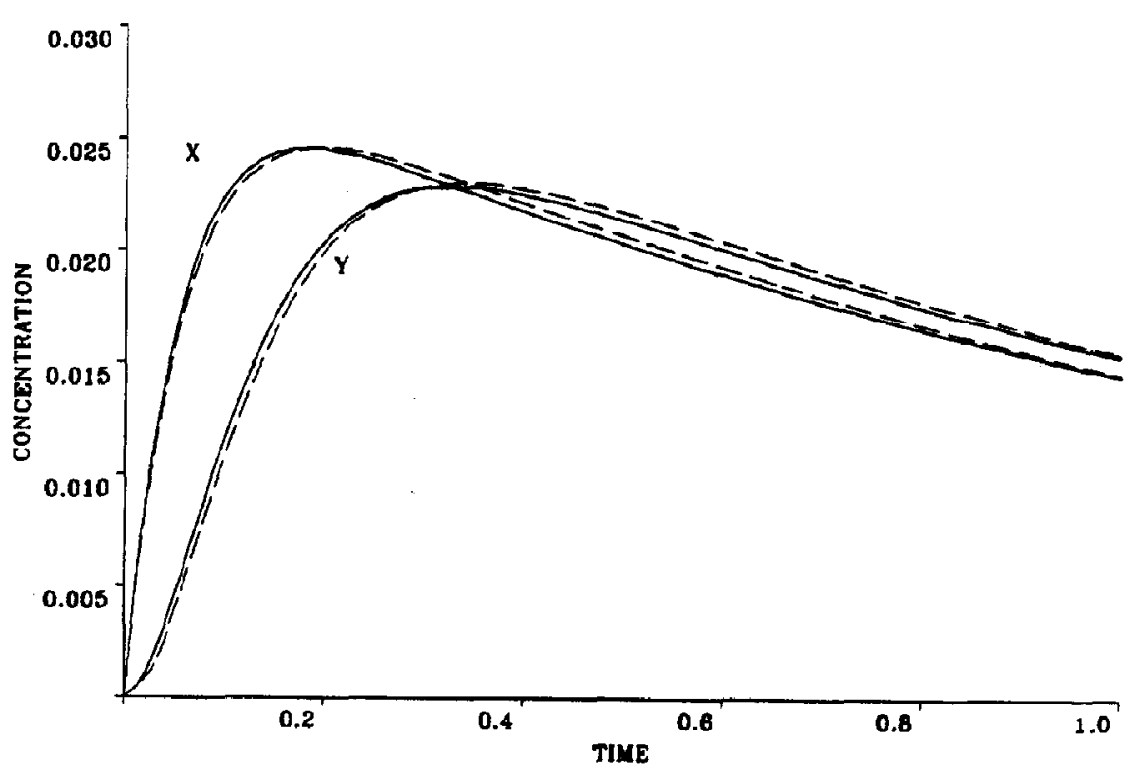

Fig. 4. Case 2 composite $(---)$ solutions compared to numerical solutions $(-X$ ) for $X$ and $Y$, the intermediate concentrations. Parameters are the same as in fig. 1. 
solution set were used, and small terms were kept to give

$$
\begin{aligned}
e_{\text {icomp }} & =\epsilon \frac{q p}{\psi}\left(1-e^{-t /\left(t_{s}(1+\rho)\right)}\right) \\
& +\epsilon^{2} q p\left[\frac{e^{-t / t_{s}}}{(1+\rho)(\psi-1)}-\frac{e^{-\psi t / t}}{\psi^{2}(\psi-1)(1+\rho)}\right. \\
& +e^{-t /\left(t_{s}(1+\rho)\right)}\left(-\frac{1+p+\psi}{\psi^{2}(1+\rho)}-\frac{p}{\psi}+\frac{q p}{\psi^{2}}\right) \\
& +e^{-2 t /\left(t_{s}(1+\rho)\right)}\left(\frac{p}{\psi^{2}(1+\rho)}+\frac{p}{\psi}-\frac{q p}{\psi^{2}}\right) \\
& \left.+\frac{t}{t_{s}} e^{-t /\left(t_{s}(1+\rho)\right)}\left(-\frac{q p}{\psi^{2}(1+\rho)}-\frac{\rho}{\psi(1+\rho)^{2}}\right)\right]
\end{aligned}
$$

These graphs illustrate that the composite solutions are far more accurate than previous solutions in the inner domain.

Fig. 4 shows the numerical solutions compared to the composite solutions for $X$ and $Y$. The first term of the case 2 composite solution as previously given was used for each of $X$ and $Y$. These intermediate results are much more accurate than any pseudo-steady state method has been able to achieve, since the present method incorporates the variation of the intermediate time derivatives prior to the pseudo-steady state.

\section{Discussion}

The above results show that the solutions described here are a valid approximation of the kinetics of the suicide substrate system. The solutions for $S$ and $E_{\mathrm{i}}$ are more accurate than those of previous attempts $[5,6]$ for $E_{0} / S_{0}=O(1)$, especially in the inner domain (small time), which is by definition ignored by any pseudo-steady-state method. It should be noted that solutions may be derived for $E_{0} / S_{0}$ of other magnitudes by the same method. Moreover, the method developed here is particularly useful in estimating the intermediate $(X$ and $Y$ ) concentrations which no previous analysis has been able to do. Perhaps the most important result of the method described here is that solutions are obtained analytically in terms of the kinetic parameters. These solutions may be used to estimate the parameters by the methods described by Waley [14] and Duggleby [7]. Such analytical solutions are especially important when the equations are stiff, i.e., when small parameters multiply derivatives in the differential equation system, when numerical solutions are notoriously difficult to compute accurately.

\section{Acknowledgements}

J.D.M. dedicates this article to his long-time friend, colleague and mentor, Jeffries Wyman, who was primarily responsible for J.D.M. becoming a mathematical biologist. This material is based upon work supported under a National Science Foundation Graduate Fellowship. We would especially like to thank Dr. Stephen Waley for several helpful discussions. M.A.B. would like to acknowledge the Universities of the United Kingdom Overseas Research Scheme, and P.K.M. would like to thank the Science and Engineering Research Council of Great Britain for its support through a grant to the Centre for Mathematical Biology. Finally, M.A.B. wishes to thank the University of Washington Applied Mathematics Department for its hospitality in 1988-89.

\section{References}

1 R. Rando, Methods Enzymol. 46 (1977) 28.

2 N. Seiler, M.J. Jung and J. Koch-Weser, Enzyme-activated irreversible inhibitors (Elsevier/North-Holland, Oxford, 1978).

3 C.T. Walsh, Annu, Rev, Biochem. 53 (1984) 493.

4 C. Walsh, T. Cromartie, P. Marcotte and R. Spencer, Methods Enzymol. 53 (1978) 437.

5 S.G. Waley, Biochem. J. 185 (1980) 771.

6 S. Tatsunami, N. Yago and M. Hosoe, Biochim. Biophys. Acta 662 (1981) 226.

7 R.G. Duggleby, J. Theor. Biol. 123 (1986) 67.

8 L.A. Segel and M. Slemrod, SIAM Rev. 31 (1989) 446.

9 C.L. Frenzen and P.K. Maini, J. Math. Biol, 26 (1988) 689.

10 L.A. Segel, Bull. Math. Biol. 50 (1988) 579.

11 J.D. Murray, Asymptotic analysis (Springer, Heidelberg, 1984) p. 138.

12 J.D. Murray, Mathematical biology (Springer, Heidelberg, 1989) p. 113.

13 J. Kevorkian and J.D. Cole, Perturbation methods in applied mathematics (Springer, Heidelberg, 1981) p. 7.

14 S.G. Waley, Biochem. J. 227 (1985) 843. 